Background $\mathrm{CDH}$ was the most common cause of death in newborn group of surgical patients in our hospital. Statistics significantly changed after introducing the delayed approach of surgical correction.

Objectives Study objectives are to assess the effectiveness of delayed surgical correction in patients with $\mathrm{CDH}$.

Methods We compared Two groups of patients with $\mathrm{CDH}$, who underwent the surgery. In the first group, since 1978, great majority of patients were operated in first $24-48 \mathrm{~h}$ of life. In second group, since 2007, time of surgical intervention was approximately at $\mathrm{t} 98 \mathrm{~h}$ after birth. In 98\%, in both groups, diaphragmatic defect was primarily closed. Only in $2 \%$ of cases patch or silo was used. None extra corporal membrane oxygenation or preoperative NO was never used.

Conclusion Delayed surgical correction $-96 \mathrm{~h}$ after birth, after initial management and pulmonary support, improves the outcome in patients with $\mathrm{CDH}$, compared with surgical intervention in first 24-48 h of life.

\section{PS-295 CLINICAL EXPERIENCE IN APPLICATION OF THORACOSCOPY IN TREATMENT OF CONGENITAL DIAPHRAGMATIC HERNIA IN NEWBORNS}

${ }^{1}$ A Svirsky, ${ }^{1} \mathrm{~V}$ Averin, ${ }^{2} \mathrm{~A}$ Mahlin, ${ }^{3} \mathrm{~A}$ Svirskaya. ${ }^{1}$ Pediatric Surgery, Belarusian State Medical University, Minsk, Belarus; ${ }^{2}$ Pediatric Surgery, Pediatric Surgery Centre, Minsk, Belarus; ${ }^{3}$ NICU, Republican Scientific and Practical Centre "Mother and Child", Minsk, Belarus

10.1136/archdischild-2014-307384.593

Background and aims The treatment of newborns with congenital diaphragmatic hernia $(\mathrm{CDH})$ is still actual problem. Changing management tactics has led to an improvement in results last decade. The mini-invasive surgical procedure was developed and introduced in clinic adding new abilities.

The goal is to determine the effectiveness of thoracoscopy in treatment of $\mathrm{CDH}$ in newborns.

Methods The retrospective analysis of treatment of 127 infants with CDH during the period from 1993 to 2013 in Paediatric Surgery Centre of Minsk, Belarus was conducted. From 1993 to 2006, the newborns were operated on 1-2 day admission. Traditional methodslaparotomy and thoracotomy applied in 59 of 65 children in this group, 16 patients died. Post operativelethality was $27.1 \%$, the overall mortality rate $30.7 \%$. Postoperative complications have demanded 18 (30.5\%) reoperations in 13 patients, including 4 hernias relapse. From January 2007 operation are produced only in children with stable condition on the 6-8 day of life. The main method from December 2009 is thoracoscopic plastic. The 62 children have been treated. Operations were performed in 54 children: 16 children by traditional methods and 38 completed mini-invasive intervention.

Results Four patients (7.4\%) died postoperatively and overall mortality amounted to $12.9 \%$. Reoperations took place in 10 patients (18.6\%), 5 children have recurrent hernia after thoracoscopic plastic. One child relapse marked twice in 6 months. All children repeatedly operated with thoracoscopy, in four cases patches were applied. No complications were revealed postoperatively.

Conclusion Thoracoscopy for the treatment of $\mathrm{CDH}$ is effective method.
PS-296

HIRSCHSPRUNG'S DISEASE PRESENTING IN THE NEONATAL PERIOD: A REGIONAL CENTRE EXPERIENCE

${ }^{1} \mathrm{R}$ Zogopoulou, ${ }^{1} \mathrm{~S}$ Kumar, ${ }^{2} \mathrm{~A}$ Mudher, ${ }^{3} \mathrm{M}$ Agrawal, ${ }^{1} \mathrm{~A}$ Kapetanakis. ${ }^{1}$ Neonatal Medicine, Evelina London Children's Hospital, London, UK; ${ }^{2}$ Histopathology, St Thomas Hospital, London, UK; ${ }^{3}$ Paediatric Surgery, Evelina London Children's Hospital, London, UK

10.1136/archdischild-2014-307384.594

Background Hirschsprung Disease (HD) is characterised by the absence of ganglion cells in the distal bowel commonly presents in neonatal period with a suggestive history. However a rectal biopsy $(\mathrm{RB})$ is required to confirm the diagnosis. The reported incidence in the UK is 1:5000.

Aim To describe the clinical characteristics of neonates with HD presenting to a regional neonatal surgical centre.

Methods Retrospective review of clinical records over a 2 year period (2012-2013).

Results $15 / 51$ infants evaluated had a positive rectal biopsy (RB) 4 infants were inborn (Inborn Incidence 1/3000), 11 were referred from 7 other London hospitals.

Initial presenting complaint in the HD (non HD) groups was: Abdominal distention 93\% (95\%) Delayed passage of meconium $78.5 \%,(69.5 \%)$ Bilious vomiting 57\% (82\%), Poor feeding 7\% (35\%).

Gestation age (mean,(range)) was 39 (37-41). Infants were referred on $\mathrm{d}^{3}(1-10) \mathrm{RB}$ was performed on day 9 (2-37), $85 \%$ were initially managed with rectal washouts and discharged home. 2 infants required an operation in the neonatal period. The remainder underwent surgical procedure on day 159(61521).

Conclusion HD needs to be considered and a RB performed in infants with consistent history and examination Especially abdominal distention or history of delayed passage of meconium. The incidence of HD may be higher in some populations and this should be evaluated further.

\section{PS-297 IMPROVING QUALITY OF CARE IN CONGENITAL DIAPHRAGMATIC HERNIA (CDH)}

${ }^{1}$ A Awoseyila, ${ }^{2} \mathrm{~V}$ Baral. ${ }^{1}$ Neonatology, Southampton University Hospital Trust, Southampton, UK; ${ }^{2}$ Neonatology, Singapore General Hospital, Singapore 169856, singapore

\subsection{6/archdischild-2014-307384.595}

Aims An observation of a high mortality over 12 months (83.3\% in 2012) amongst infants with $\mathrm{CDH}$ prompted a retrospective review of management to evaluate outcomes over the past 5 years and compare mortality rates with the previous 5 year block. Care pathways were reviewed to see if variations in management existed within the team.

Method Data was collected retrospectively from case records as well as from foetal medicine and Neonatal Databases (January 2007-December 2012).

Results 53 cases of antenatal CDH were identified. Termination of pregnancy was performed in 15/53 (28.3\%) and intrauterine death occurred in 1 case. 2 cases were excluded due to incomplete data (including 1 live birth). There were 36/53 live births (68\%). 11/35 (31.4\%) had an associated major anomaly. Survival to discharge rate for isolated $\mathrm{CDH}$ was $14 / 24$ (58.3\%) compared with $3 / 11(27.3 \%)$ in the anomaly group. Overall survival to discharge rate of live births was $17 / 36$ (47.2\%). 2 infants received 
ECMO, both of whom survived to discharge as did all babies who underwent surgical repair of the $\mathrm{CDH}$.

Conclusion There was an $11.1 \%$ mortality rate increase amongst the cohort studied when compared with the preceding 5 year block. A notably lower termination rate (28\% vs. 50\%) could possibly account for this, in addition to associated anomalies as above. Variations in management approach within the team was observed leading to the subsequent formulation of an evidence based protocol to improve care quality and future outcomes as current evidence suggests.

\section{PS-297a CATCH-UP-GROWTH IN TERM AND PRETERM INFANTS AFTER SURGICAL CLOSURE OF VENTRICULAR SEPTAL DEFECT IN THE FIRST YEAR OF LIFE: ONLY GOOD NEWS?}

${ }^{1} \mathrm{~L}$ Martins, ${ }^{1} \mathrm{R}$ Lourenço, ${ }^{2} \mathrm{~S}$ Cordeiro, ${ }^{2} \mathrm{~N}$ Carvalho, ${ }^{3} \mathrm{M}$ Loureiro, ${ }^{3} \mathrm{M}$ Patrício, ${ }^{2} \mathrm{R}$ Anjos. ${ }^{1}$ Department of Pediatrics, Hospital Do Divino Espirito Santo de Ponta Delgada EPE, Ponta Delgada, Portugal; ' 2 Department of Pediatric Cardiology, Hospital de Santa Cruz Centro Hospitalar de Lisboa Ocidental EPE, Lisbon, Portugal; ${ }^{3}$ Laboratory of Biostatistics and Medical Informatics IBILI, Faculty of Medicine of University of Coimbra, Coimbra, Portugal

\subsection{6/archdischild-2014-307384.596}

Failure to thrive is common in children with non-restrictive ventricular septal defect (VSD). Normalisation of growth has been reported after early surgical correction. However the literature is inconsistent about growth velocity after surgery in term and preterm infants.

Objective Establishing the pattern of catch-up growth in infants submitted to VSD surgical repair before 1 year of age, for term and preterm infants.

Methods 52 infants (41 term, 11 preterm) were studied. Anthropometric data at birth, at time of surgery and 3, 6, 12 and 24 months after surgery, collected retrospectively, were converted to z-scores. Statistic analyses was performed in SPSS ${ }^{\circledR}$ version 21, $\alpha=0,05$.

Results Mean weight and height $\mathrm{z}$ scores at the time of surgery were significantly lower for term infants $(-2,24$ and $-1,42$, respectively; $\mathrm{p}<0,001)$ and preterm infants $(-3,07$ and $-2,22 ; \mathrm{p}$ $=0,003)$. A higher growth velocity was observed in the first three months after surgery. For term infants, catch-up growth was completed 6 months after surgery (mean weight and height z scores were $-0,39$ and $-0,7$, respectively). Preterm infants completed their catch up growth one year after surgery. There were no statistically significant differences in mean weight and height between term and preterm infants 24 month after surgery.

Conclusions Early surgical repair of VSD leads to a significant acceleration of growth, mainly in the first 3-6 months after surgery. An increased weight gain velocity has been associated with higher cardiovascular risk later in life. Knowledge of this specific
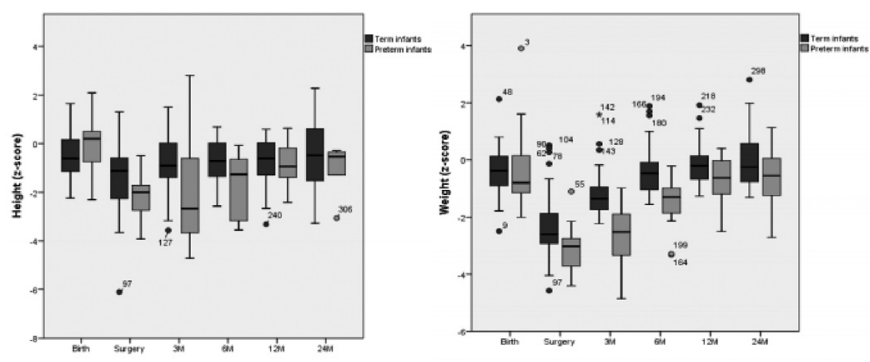

Abstract PS-297a Figure 1 catch-up growth pattern is important and should influence nutritional goals after surgery.

\section{Perinatal Infection}

\section{PS-298 RANDOMISED CONTROLLED TRIAL TO COMPARE EFFICACY OF DIFFERENT TIMING OF ANTIBIOTICS AT CAESAREAN SECTION AND THEIR EFFECT ON MOTHER AND NEWBORN}

${ }^{1}$ KA Kuruvilla, ${ }^{1} \mathrm{CA}$ Jyothirmayi, ${ }^{2} \mathrm{~A}$ Halder, ${ }^{2} \mathrm{R}$ Jose, ${ }^{1} \mathrm{~S}$ Sridhar. ${ }^{1} \mathrm{C}$ hild Health and Neonatology, Christian Medical College, Vellore, India; ${ }^{2}$ Obstetrics and Gynaecology, Christian Medical College, Vellore, India

\subsection{6/archdischild-2014-307384.597}

Background During caesarean section, prophylactic antibiotics are usually given after cord clamping (instead of prior to skin incision) for fear of antibiotics having adverse effects on the newborn and promoting resistant strains.

Objective To compare efficacy of intravenous cefazoline administered during caesarean section either before skin incision or after cord clamping, on mother and newborn.

Setting Tertiary care perinatal centre in south India.

Methods Term gestation mothers posted for caesarean section were randomised to receive two medicinesIV cefazoline/ placeboone prior to skin incision, one after cord clamping. Mothers and babies were monitored for evidence of infection or adverse events during hospital stay. They were reviewed at 45 days to look for complications.

Results 1106 mothers were recruited. At baseline, mothers and babies in both groups were similar. The mean (SD) duration of hospital stay for mothers in both groups was 5.3(1.5) days. Mothers who received antibiotics prior to skin incision had less post operative complications compared to mothers who received antibiotics after cord clamping $(p=0.000)$. Mothers who received antibiotics after cord clamping stayed longer in hospital $(\mathrm{p}=0.008)$. Babies in both groups had similar rates of nursery admissions, sepsis, NEC and hospital readmission following discharge.

Conclusions IV antibiotics can be safely administered to mothers prior to skin incision which decreases postoperative infectious morbidity without adverse effects in babies.

\section{PS-299 HAEMATOLOGICAL MARKERS OF VERTICAL TRANSMISSION OF GENITAL MYCOPLASMAS IN PREMATURE INFANTS}

${ }^{1} \mathrm{~N}$ Hussain, ${ }^{2} \mathrm{~L}$ Rahman. 'Pediatrics, Connecticut Children's Medical Center, Hartford, USA; ${ }^{2}$ Pediatrics, University of Connecticut Health Center, Farmington, USA

\subsection{6/archdischild-2014-307384.598}

Background Genital mycoplasmas (Ureaplasma urealyticum-Uu or Mycoplasma hominis - Mh) are low grade pathogens associated with complications of pregnancy (chorioamnionitis and preterm labour); but their role as neonatal pathogens is controversial.

Aim To identify haematological markers of vertical transmission of genital mycoplasma in premature newborn infants.

Methods A retrospective cohort study done at University of Connecticut Health Centre NICU with admissions from 20032010. Intubated infants in the NICU had tracheal cultures sent 